\title{
An Adaptive Root-Solving Controller for Tracking of Nonlinear Dynamic Plants
}

\author{
Naveed R. Butt, Muhammad Shafiq and Tahir Khan
}

\begin{abstract}
Adaptive tracking of nonlinear dynamic plants is currently an important area of research. The main difficulty being felt by the research community is the lack of a general modelling framework that can facilitate synthesis of a simplistic control law, while being capable of providing accurate approximation of nonlinear systems. In this study a novel technique based on the control-oriented $U$-model is proposed for the adaptive tracking of a wide range of stable nonlinear dynamic plants using only past data. The basic scheme is based on the robust internal model control (IMC) structure. An adaptive version of the U-model is introduced for the modelling of the plant and a simplistic control law based on polynomial root-solving is synthesized. The proposed scheme has a more general appeal than other adaptive schemes based on models such as the polynomial NARMAX (Nonlinear Autoregressive Moving Average with Exogenous inputs) model. Additionally, the control law is much simpler in nature. The effectiveness of the proposed adaptive scheme is demonstrated through application to the real-time control of a single-link robotic arm manipulator and simulations for different nonlinear plants.
\end{abstract}

\section{INTRODUCTION}

The primary objective of controller design is to influence the behavior of systems. A typical control objective is tracking which involves forcing system variables to follow prescribed trajectories. Today, some of the main difficulties to be overcome by the designer are the nonlinear plant dynamics and the uncertainties caused by differences between actual and assumed dynamic models. Most physical systems are nonlinear and multivariate. By nature, they have inherent interconnected nonlinearities in their dynamics where the relationship between the input and output variables varies depending on the operating conditions. If the nonlinearities are mild or the operating conditions do not change much, then the effect of the nonlinearities is not severe, and linear control techniques are applicable. However, many industrial systems exhibit strong nonlinear behavior and they may be required to operate over a wide range of operating conditions. Additionally, there are situations where the nonlinear plants are extremely difficult to model and/or they exhibit high uncertainties. Under these conditions, the conventional constant gain feedback controller fails to maintain the performance

Research supported by King Fahd University of Petroleum and Minerals, Dharan, Saudi Arabia

The authors, N. R. Butt ( nrbutt@ccse.kfupm.edu.sa), M. Shafiq ( mshafiq@ecse.kfupm.edu.sa) and T. Khan ( tahir@ccse.kfupm.edu.sa) are with the Systems Engineering Department, College of Computer Science and Engineering, King Fahd University of Petroleum and Minerals, Dhahran, Saudi Arabia. of the system at acceptable levels and does not respond well to changes in system dynamics. This has resulted in an increased interest in developing controllers whose parameters can vary online (thus adapting to the current plant dynamics).

Adaptive tracking of uncertain nonlinear dynamic plants has become an important area of research and several adaptive control designs have been recently developed. An extensive discussion on most of these designs can be found in [1]. In particular, model based control (MBC) strategies form an important part of adaptive control theory and generally involve two important choices

1) Selection of an identification scheme

2) Selection of a control law based on the structure of the identifying scheme.

It is clear that the modelling part plays a very important role in the overall system design and performance. This is because the control law is actually based upon the model structure. Two of the more important measures of model utility are its approximation capabilities (in terms of accuracy) and suitability for control (in terms of computational ease). It has been pointed out in literature [2], [3] that the main difficulty in the area of adaptive nonlinear control is the lack of a general modelling framework that satisfies both of the above mentioned measures simultaneously. For instance, NARMAX (Nonlinear Autoregressive Moving Average with Exogenous inputs) the most commonly used nonlinear modelling structure has the ability to represent a broad range of nonlinear systems [4]. However because of its overly complex structure, it does not lend itself to easy manoeuvring for controller design [5].On the other hand, linear models, that are easy to maneuver, are inherently incapable of describing an enormous range of important dynamic phenomena.

This paper deals with the genuine need for a model based tracking scheme for nonlinear plants that is general, sufficiently accurate and enables the synthesis of a simplistic control law. The key idea is based on the recently proposed control-oriented nonlinear model termed as the U-model [2]. The U-model has a more general appeal as compared to the polynomial NARMAX model [6] and Hammerstein model. Additionally, this model is control-oriented in nature which makes the control synthesis part easier. Based on the Umodel a pole placement controller [2] and a new IMC (Internal Model Control) structure for dynamic nonlinear plants with known parameters [7] have recently been proposed. This paper introduces an adaptive IMC scheme based on the U-model utilizing nonlinear adaptive filtering. The main advantages of the proposed approach are its generality and 


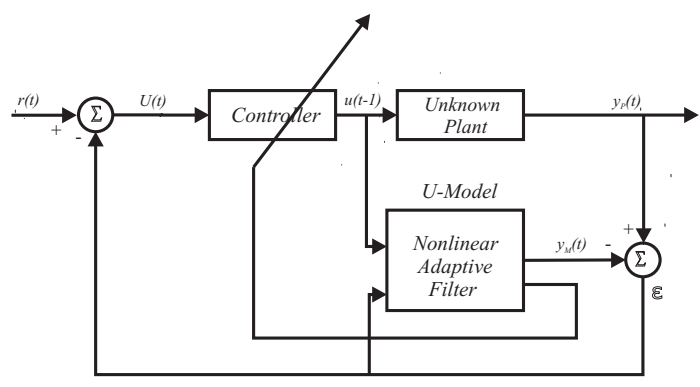

Fig. 1. Proposed Structure Block Diagram

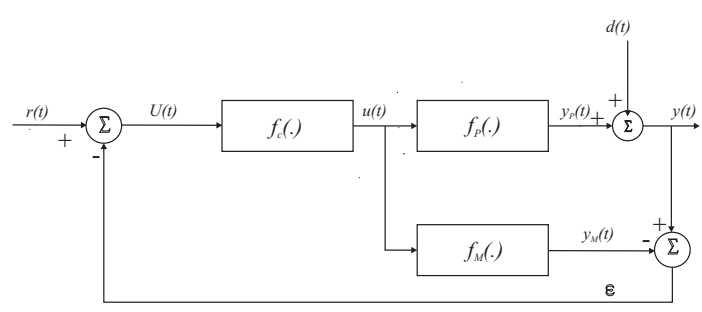

Fig. 2. IMC Block Diagram

simplistic control law.

The proposed structure is depicted in the block diagram (figure 1), where the dynamic nonlinear plant is modelled as a U-model whose parameters are identified using nonlinear adaptive filter and are used to synthesize a simple law for control inputs to the plant.

The rest of the paper has been organized as follows. Section II presents a brief introduction to the IMC, the U-Model and nonlinear adaptive filtering. A new adaptive IMC based on the U-model is proposed in section III. The effectiveness of the proposed scheme is verified through simulations and real-time implementation for tracking of various nonlinear plants. These are discussed in sections IV and $\mathrm{V}$, respectively.

\section{PRELIMINARIES}

\section{A. Internal Model Control}

IMC is one of the most popular control strategies used in industrial process control. Its main features are its simple structure, fine disturbance rejection capabilities and robustness [8]-[13]. IMC can be used for both linear and nonlinear systems [14] and is especially suitable for the design and implementation of open-loop stable systems. Many industrial processes happen to be intrinsically open-loop stable. Figure 2 depicts the basic structure of the IMC.

Here the plant is represented by $f_{P}($.$) , the model by$ $f_{M}($.$) and the controller by f_{C}($.$) . The symbols r(t), u(t)$ and $d(t)$ represent the reference input, the control signal and the additive noise respectively. A detailed analysis of the properties of IMC has been given in [15]. Important characteristics of the IMC are summarized with the following properties.

1) Property $P 1$ (Dual Stability): If the plant and the controller are input-output stable and the model is a perfect representation of the plant; then the closed-loop system is input-output stable.

2) Property P2 (Perfect Control): If the inverse of the operator describing the plant model exists, and this inverse is used as the controller, and the closed-loop system is input-output stable with this controller; then the control will be perfect.

3) Property P3 (Zero Offset): If the inverse of the steady state model operator exists, and the steady state controller operator is equal to this, and the closed-loop system is input-output stable with this controller. Then offset free control is attained for asymptotically constant inputs.

\section{B. The U-Model}

The control-oriented U-model plays a central role in the adaptive scheme proposed in this paper. Following is the development of the U-model based on [2]. Consider singleinput single-output (SISO) nonlinear dynamic plant with a NARMAX representation of the form:

$$
\begin{gathered}
y(t)=f[y(t-1), \ldots, y(t-n), u(t-1), \ldots, \\
u(t-n), e(t), \ldots, e(t-n)]
\end{gathered}
$$

where $y(t)$ and $u(t)$ are the output and input signals of the plant respectively at discrete time instant $t, n$ is the order of the plant, $f($.$) is a nonlinear function and e(t)$ represents the error due to measurement noise, model mismatch, uncertain dynamics and plant variation. The U-model is obtained by expanding the nonlinear function $f($.$) of the above equation$ as a polynomial with respect to $u(t-1)$ as follows:

$$
y(t)=\sum_{j=0}^{M} \alpha_{j}(t) u^{j}(t-1)+e(t)
$$

Where $M$ is the degree of model input $u(t-1), \alpha_{j}(t)$ is a function of past inputs and outputs $u(t-2), \ldots, u(t-$ $n), y(t-1), \ldots, y(t-n)$ and errors $e(t-1), \ldots, e(t-n)$ To apply linear control system design methodologies to the nonlinear model a further transformation is applied as follows:

$$
y(t)=U(t)
$$

Where,

$$
U(t)=\Phi[u(t-1)]+e(t)=\sum_{j=0}^{M} \alpha_{j}(t) u^{j}(t-1)+e(t)
$$

The expression (3) is defined as the U-model. This model has the following advantages:

1) The control-oriented U-model is more general than other parameterizing approaches, such as the polynomial NARMAX model [6], the Hammerstein model etc.

2) The sampled data representation of many non-linear continuous time systems can be of the form as follows:

$$
y(t)=\sum_{j=0}^{M} \alpha_{j}(t) u^{j}(t-1)
$$




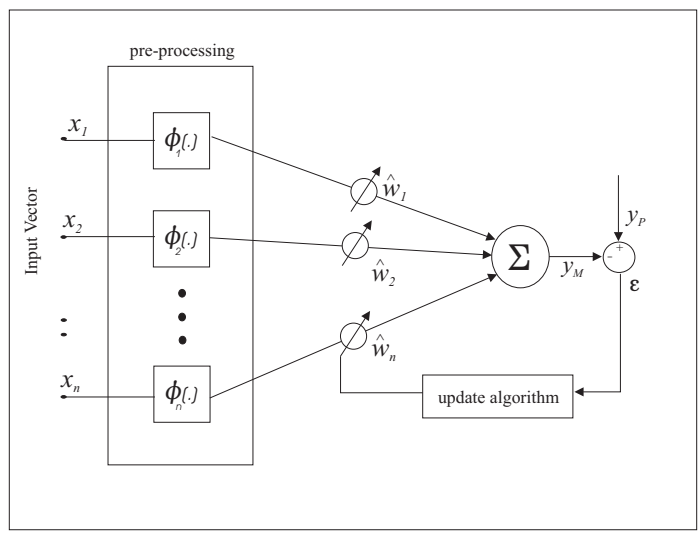

Fig. 3. General Nonlinear Adaptive filter

3) The U-model exhibits a polynomial structure in the current control $u(t-1)$.

4) Due to its polynomial structure, the nonlinear algebraic equations, which need to be solved to obtain the output value of the controller, are also polynomials in $u(t-1)$, unlike other models which lead to complex non-linear algebraic equations.

For the case of IMC presented in figure 2, the control signal $u(t-1)$ can be obtained easily by using NewtonRaphson algorithm recursively with $U(t)$ (as defined in figure) as a root solver.

$$
\begin{aligned}
& u_{i+1}(t-1)= \\
& u_{i}(t-1)-\left.\frac{\sum_{j=0}^{K} \alpha_{j}(t) u_{i}^{j}(t-1)-U(t)}{d\left[\sum_{j=0}^{K} \alpha_{j}(t) u^{j}(t-1)\right] / d u(t-1)}\right|_{u^{j}(t-1)=u_{i}^{j}(t-1)}
\end{aligned}
$$

where the subscript $i$ is the iteration index [2]. Thus the problem of finding the inverse of the plant has been effectively reduced to an iterative root-solving procedure.

\section{Nonlinear Adaptive Filter}

Accurate estimation of the plant is an important part of the IMC scheme. Adaptive filters with nonlinear preprocessing of the inputs provide a versatile structure for the estimation task. It is therefore proposed to use a nonlinear adaptive filter of the form depicted in fig.3. Here the input variables $\left(x_{1}, x_{2}, \ldots, x_{n}\right)$ are pre-processed through nonlinear functions $\phi_{i}($.$) . These are then input to the adaptive$ linear combiner that adaptively assigns weights to these inputs. The resulting sum gives the approximated output which can be written as

$$
y_{M}=\sum_{i=1}^{n} w_{i} \phi_{i}\left(x_{i}\right)
$$

A proper selection of the nonlinear functions $\phi_{i}($.$) is impor-$ tant for the overall performance of the structure [16]. The weights are updated iteratively using the normalized Least Mean Square (nLMS) algorithm. This algorithm seeks to minimize the mean squared error $E\left\{\left|y_{P}-y_{M}\right|^{2}\right\}$, where $y_{P}$ is the desired output.

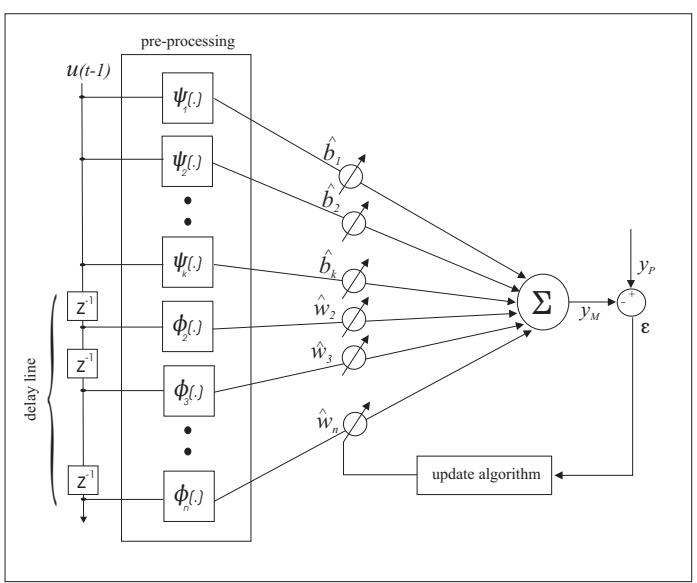

Fig. 4. Proposed Nonlinear Adaptive filter

\section{U-MODEL BASED ADAPTIVE IMC USING NONLINEAR ADAPTIVE FILTER}

This section proposes a new adaptive IMC based on Umodel for tracking of nonlinear dynamic plants with using only the past inputs and outputs. In the following, reference is made to figure 1 .

\section{A. The Plant}

The scheme assumes a stable nonlinear dynamic plant whose functional parameters or the functional structure need not be known.

\section{B. The Identifying Model}

It is proposed to identify the plant online using the nonlinear adaptive filter depicted in figure 4 . Here the model output is given by

$$
\begin{aligned}
y_{M}(t) & =\hat{b}_{1} \psi_{1}(u(t-1))+\hat{b}_{2} \psi_{2}(u(t-1)) \\
& +\ldots+\hat{b}_{k} \psi_{k}(u(t-1))+\hat{w}_{2} \phi_{2}(u(t-2)) \\
& +\hat{w}_{3} \phi_{3}(u(t-3))+\ldots+\hat{w}_{n} \phi_{n}(u(t-n))
\end{aligned}
$$

In order to make this structure suitable for use with the Umodel the functions $\psi_{i}($.$) are defined to represent the powers$ of the current input signal $u(t-1)$. i.e.,

$$
\psi_{i}(u(t-1))=u^{i}(t-1) \quad i=1,2, \ldots, k
$$

Additionally, it is proposed to use gaussian radial basis function $(\mathrm{RBF})$ to represent the remaining nonlinear functions $\phi_{j}($.$) in eq. 5$.

$$
\phi_{j}(u(t-j))=e^{-\left(\frac{u(t-j)-m_{j}}{c_{j}}\right)^{2}} \quad l, 3, \ldots, n
$$

where $m_{i}$ and $c_{i}$ represent the center and spread parameters of the gaussian RBF respectively. Radial basis functions are among the most commonly used functions in neural networks 
and have good approximation capabilities [17]. With these selections the model (eq.5) can now be written as

$$
\begin{aligned}
y_{M}(t) & =\hat{b}_{1} u(t-1)+\ldots+\hat{b}_{k} u^{k}(t-1) \\
& +\hat{w}_{2} \phi_{2}(u(t-2))+\ldots+\hat{w}_{n} \phi_{n}(u(t-n))
\end{aligned}
$$

where the functions $\phi_{i}($.$) are given by eq. 7$.

This structure has been specially tailored for use with the U-model, where the first $k$ terms represent powers of the current input and the last $n-1$ terms represent the contributions due to past data. The number of terms required in the model (eq.8) can be adjusted to best suit the plant at hand. As suggested in section II-C all the weights are adjusted using nLMS algorithm.

\section{The Control Law}

To allow for the synthesis of a simplistic control law, eq.8 is converted into its equivalent U-model

$$
y_{M}(t)=\sum_{j=0}^{N} \hat{\alpha}_{j} u^{j}(t-1)
$$

where

$$
\hat{\alpha}_{0}=\hat{w}_{2} \phi_{2}(u(t-2))+\ldots+\hat{w}_{n} \phi_{n}(u(t-n))
$$

and

$$
\hat{\alpha}_{1}=\hat{b}_{1}, \hat{\alpha}_{2}=\hat{b}_{2}, \ldots
$$

With this structure the control inputs can be easily obtained using the Newton-Raphson root solving algorithm given by eq.4. It must be noted that the proposed scheme leads to a very simple and general control law. This approach is therefore expected to prove extremely useful in the area of nonlinear control.

\section{System Operation}

As shown in figure 1 , the output of the controller $u(t)$ is fed to both the unknown plant and the nonlinear adaptive filter. The mismatch error $\varepsilon$ input to the filter is the difference between the output of the plant $y_{P}(t)$ and the output of the radial basis nonlinear moving average filter $y_{M}(t)$. The filter parameters are updated using normalized least mean square algorithm such that the error $\varepsilon$ is minimized. A copy of the filter parameters which are the parameters of the U-model is fed to the controller online and the controller calculates the inverse of the unknown plant using the Newton-Raphson method based on the U-model of the plant. If the plant to be controlled is unstable then it is first stabilized using simple feedback.

There are two problems that may occur while using the Newton-Raphson root solving algorithm. First, the denominator in eq.4 may tend to zero near the solution. Second, the polynomial formed by eq. 9 may not have a real solution. To solve these problems an improved computation for the Newton-Raphson algorithm has been suggested in [18]. With these adjustments, the proposed scheme presents a quite general framework for adaptive tracking of nonlinear dynamic plants.

\section{APPLICATION TO NONLINEAR PLANTS: SIMULATIONS}

To demonstrate the application of the proposed scheme, simulations were carried out for the adaptive tracking of two nonlinear plants. These are the Hammerstein model and the continuous stirred tank reactor (CSTR). All programs were run using the Simulink platform. This section presents the results of these simulations.

\section{A. Adaptive tracking of Hammerstein model}

For the purpose of simulations the following Hammerstein model was used to serve as the unknown plant

$$
\begin{aligned}
& y(t)=0.5 y(t-1)+x(t-1)+0.1 x(t-2) \\
& x(t)=1+u(t)-u^{2}(t)+0.2 u^{3}(t)
\end{aligned}
$$

An adaptive model based on eq. 8 was used to represent the plant,

$$
\begin{array}{r}
y_{M}(t)=\hat{b}_{1} u(t-1)+\hat{b}_{2} u^{2}(t-1)+\hat{b}_{3} u^{3}(t-1) \\
+\hat{w}_{2} \phi_{2}(u(t-2))+\hat{w}_{3} \phi_{3}(u(t-3))
\end{array}
$$

and then its equivalent U-model (9) was used to synthesize the control law (4). An arbitrary reference input was selected and a sampling period of $10 \mathrm{~ms}$ was used. For the nLMS, a step size of 0.8 was used and all weights were initialized randomly between 0 and 1 . The simulation results are depicted in figures 5, and 6 . The results suggest that the proposed scheme was able to identify the nonlinear plant online, and the controller provided the appropriate inverse to facilitate tracking of the reference input.

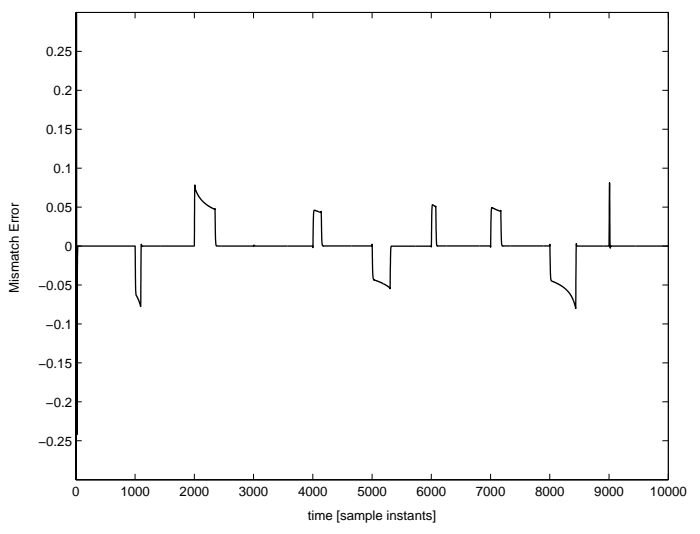

Fig. 5. Hammerstein Model: Identification error

\section{B. Adaptive tracking of CSTR}

A discrete time model for a perfectly mixed, continuous stirred tank reactor was used as the second nonlinear plant. 


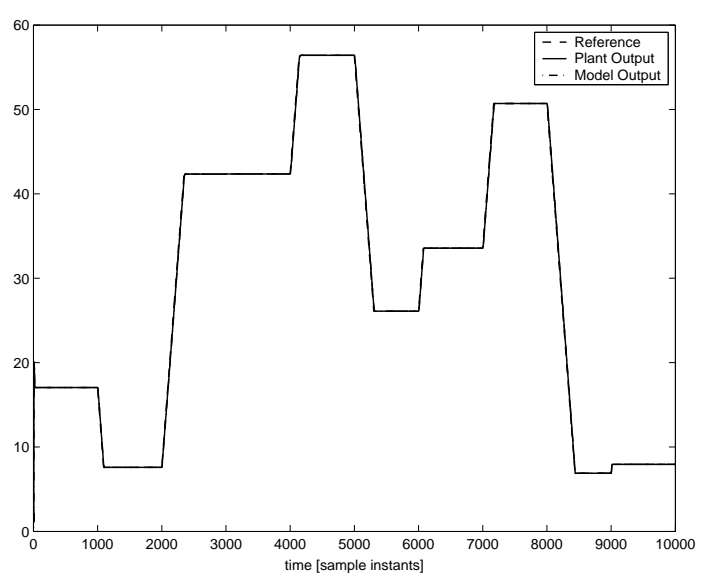

Fig. 6. Hammerstein Model: Tracking

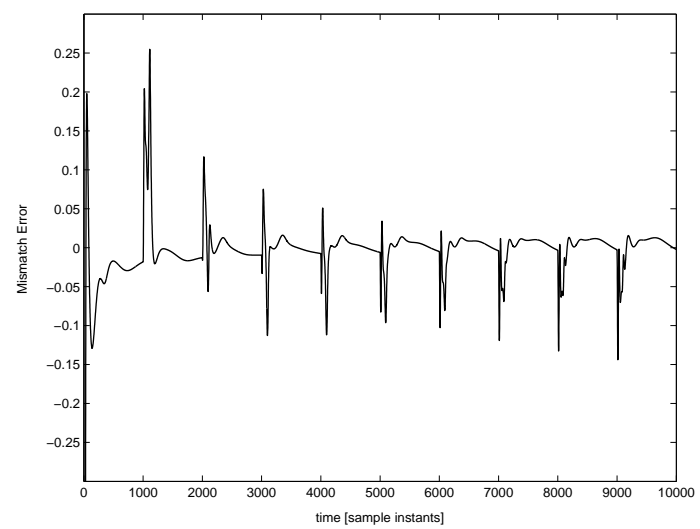

Fig. 7. CSTR: Identification error

The plant is given by [2]

$$
\begin{gathered}
y(t+1)=0.8606 y(t)-0.0401 y^{2}(t)+0.0017 y^{3}(t) \\
-0.000125 y^{4}(t)+0.0464 u(t)-0.045 y(t) u(t) \\
+0.0034 y^{2}(t) u(t)-0.00025 y^{3}(t) u(t)-0.0012 u^{2}(t) \\
+0.0013 y(t) u^{2}(t)-0.0001458 y^{2}(t) u^{2}(t) \\
+0.00002083 u^{3}(t)-0.00002083 y(t) u^{3}(t)
\end{gathered}
$$

The proposed nonlinear adaptive model (12) was used to identify the plant and the controller output was generated using the Newton-Raphson algoritm (eq.4). This time the reference input was chosen to be a periodic triangular wave. A step size of 0.5 was found suitable for this case. figures 8 and 7 show the results.

\section{REAL-TIME ADAPTIVE TRACKING OF SINGLE-LINK ROBOTIC ARM MANIPULATOR}

To further verify the applicability of the proposed scheme, experiments were carried out for the real-time adaptive tracking of a single-link robotic arm position. A block diagram explaining the setup is depicted in figure 9 where a DC-motor is used to control the position of a single-link rigid robotic arm. The brush DC-motor (Crouzet 8285002) used has a maximum speed of 3200 revolution per minute,

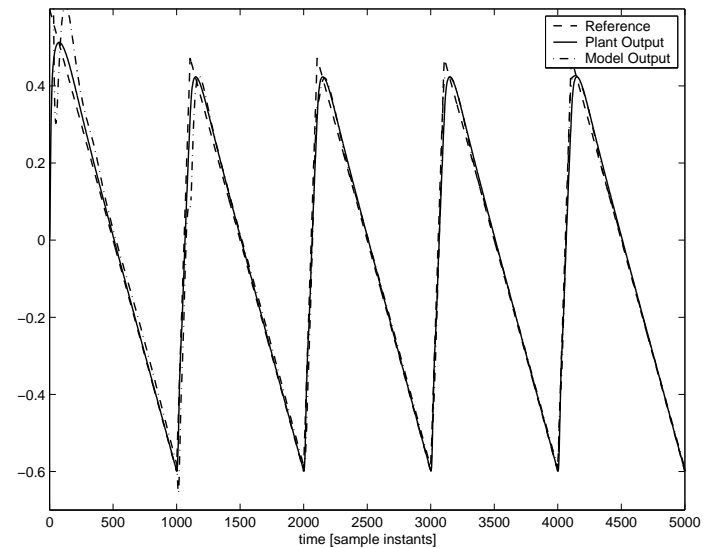

Fig. 8. CSTR: Tracking

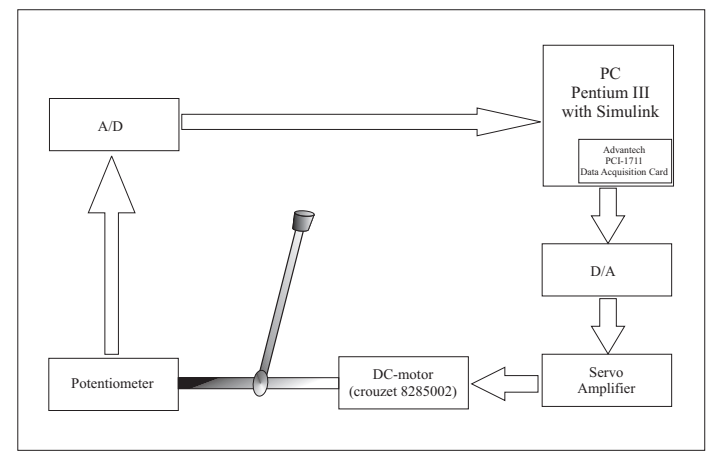

Fig. 9. Experimental Setup: Block description

which can be achieved by exciting the motor by 24 volts DC. Standard IBM PC-type Pentium III was used for the computation in real time. Data acquisition was accomplished by Advantech card PCI-1711 and the controller was implemented in Simulink real-time windows target environment. The input to the DC-motor is the voltage signal generated by the controller, while the output is the angular position of the robotic arm. This is measured using a potentiometer that gives a voltage signal proportional to the angular position of the robotic arm.

The system was first stabilized using a simple feedback controller. The complete system along with the stabilizing controller was then treated as the plant. An identification model based on eq. 8 was used to represent the plant,

$$
\begin{array}{r}
y_{M}(t)=\hat{b}_{1} u(t-1)+\hat{b}_{2} u^{2}(t-1)+\hat{b}_{3} u^{3}(t-1) \\
+\hat{w}_{2} \phi_{2}(u(t-2))+\hat{w}_{3} \phi_{3}(u(t-3))
\end{array}
$$

and then its equivalent U-model (9) was used to synthesize the control law (4). $\phi_{i}($.$) were chosen as the Gaussian radial$ basis functions. An arbitrary reference input was selected and a sampling period of $1 \mathrm{~ms}$ was used. For the nLMS, a step size of 0.5 was used and all weights were initialized randomly between 0 and 1 . The simulation results are depicted in figures 10 and 11. It is evident from this implementation that the proposed scheme was able to properly model the plant and the U-model based root-solving controller was able to 
generate appropriate control signals.

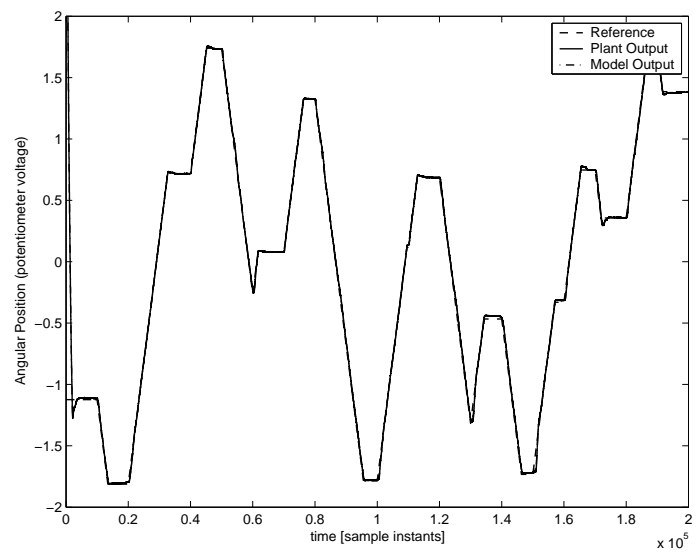

Fig. 10. Single Link Manipulator: Tracking

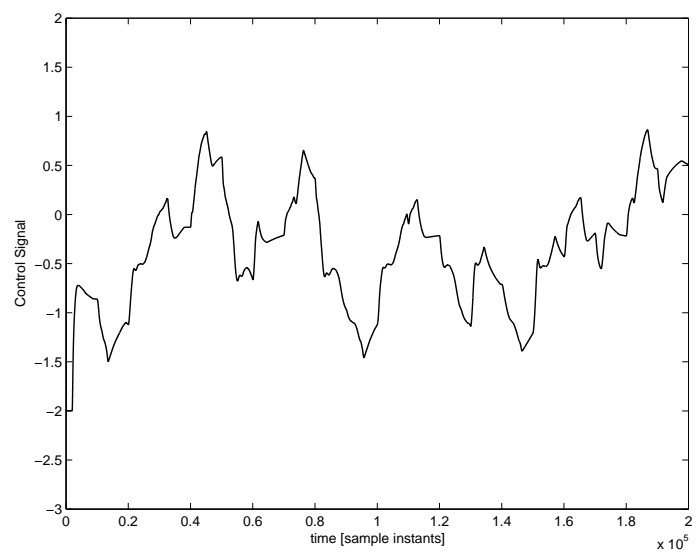

Fig. 11. Single Link Manipulator: Control signal

\section{CONCLUSIONS}

A novel technique, involving U-model based IMC, has been proposed for the adaptive control of nonlinear dynamic plants. The robustness of the IMC and the approximation power of the nonlinear adaptive filtering is combined with the control-oriented nature of the U-model to achieve adaptive tracking of stable nonlinear plants. The effectiveness of the proposed scheme is demonstrated with the help of real-time experiments and simulations. It is noted that the current contribution is expected to prove extremely useful in the area of nonlinear adaptive control.

\section{ACKNOWLEDGMENT}

The authors are grateful to the support provided by King Fahd University of Petroleum and Minerals, Dhahran, Saudi Arabia.

\section{REFERENCES}

[1] J. T. Spooner, M. Maggiore, R. Ordonez, and K. M. Passino, Stable Adaptive Control And Estimation For Nonlinear Systems. NY: WileyInterscience, 2002.

[2] Q. M. Zhu and L. Z. Guo, "A pole placement controller for nonlinear dynamic plants," J. Systems and Control Engineering, vol. 216, no. I, pp. 467-476, 2002.

[3] R. K. Pearson, "Selecting nonlinear model structures for computer control," Journal of Process Control, vol. 13, pp. 1-26, 2003.

[4] I. J. Leontarities and S. A. Billings, "Input-output parametric models for nonlinear systems. part i: Deterministic nonlinear systems; part ii: Stochastic nonlinear systems," Int. J. of Control, vol. 41, no. 2, pp. 303-344, 1985.

[5] L. Piroddi and W. Spinelli, "An identification algorithm for polynomial NARX models based on simulation error minimization." Int. J. of Control, vol. 76, no. 17, pp. 1767-1781, 2003.

[6] K. R. Sales and S. A. Billings, "Self-tuning control of nonlinear ARMAX models." Int. J. of Control, vol. 51, no. 4, pp. 753-769, January 1990

[7] M. Shafiq and M. Haseebuddin, "Internal model control for nonlinear dynamic plants using U-Model," in 12th Mediterranean Conference on Control and Automation, Turkey, June 2004.

[8] A. Datta and J. Ochoa, "Adaptive internal model control: Design and stability analysis," Automatica, vol. 32, no. 2, pp. 261-266, 1996.

[9] C. E. Garcia and M. Morari, "Internal model control.1.a unifying review and some new results," Ind. Eng. Chem. Process Des. Dev, vol. 21, pp. 308-323, 1982.

[10] S. C. Patwardhan and K. P. Madhavan, "Nonlinear internal model control using quadratic perturbation models." Computers and Chemical Engineering, vol. 22, pp. 587-601, 1998.

[11] Q. G. Wang, B. Qiang, and Y. Zhang, "Partial internal model control." IEEE Trans. On Industrial Electronics, vol. 48, no. 5, pp. 976-982, 2001.

[12] W. F. Xie and A. B. Rad, "Fuzzy adaptive internal model control." IEEE Trans. On Industrial Electronics, vol. 47, no. 1, pp. 193-202, 2000.

[13] M. Shafiq and S. H. Riyaz, "Internal model control structure using adaptive inverse control strategy." in The 4th Int. Conf. on Control and Automation (ICCA), 2003, p. 59.

[14] C. G. Economou, M. Morari, and B. O. Palsson, "Internal model control.5.extension to nonlinear systems." Ind. Eng. Chem. Process Des. Dev., vol. 25, no. I, pp. 403-411, 1986.

[15] M. Morari and E. Zafiriou, Robust Process Control. NJ: Prentice Hall, 1989

[16] B. Widrow and P. Walach, Adaptive Inverse Control. NJ: PrenticeHall, Inc., 1996.

[17] M. M. Gupta, L. Jin, and N. Homma, Static And Dynamic Neural Networks. Hoboken, NJ: John Wiley and Sons, Inc., 2003.

[18] Q. M. Zhu, K. Warwick, and J. L. Douce, "Adaptive general predictive controller for nonlinear systems," IEE Proc. Control Theory Applic., vol. 138, no. 1, pp. 33-40, 1991. 